\title{
Um catálogo de requisitos pedagógicos para auxiliar o desenvolvimento de Softwares Educacionais
}

\author{
Mychelline Souto Henrique ${ }^{1}$, Carla Taciana Lima Lourenço Silva ${ }^{2}$ \\ ${ }^{1}$ Centro de Informática - Universidade Federal de Pernambuco (UFPE) - \\ Recife - PE - Brasil \\ $\{$ msh, ctlls\}@cin.ufpe.br
}

\begin{abstract}
Studies show that the use of informatics in education enhances the process of teaching and learning, for example, Educational Software (SEs). It's known that producing SEs is not trivial, since it is necessary to identify requirements related to pedagogical perspectives. This article aims to present a viable solution to systematize the development of SEs in the requirements elicitation phase. There will be listed 62 educational requirements present in EDuCatalog4RE. The process of using the catalog will exemplify through a fictitious scenario. The evaluation of the catalog was empirical and carried by specialists. The results prove the usefulness of the catalog in the development of SES
\end{abstract}

Resumo. Estudos comprovam que o uso da informática na educação potencializa o processo de ensino e aprendizagem, um exemplo, são os Softwares Educacionais (SEs). Sabe-se que produzir SEs não é trivial, pois é preciso identificar requisitos relacionados as perspectivas pedagógicas. Este artigo tem como objetivo apresentar uma solução viável para sistematizar o desenvolvimento de SEs, na fase de elicitação de requisitos. Serão listados 62 requisitos pedagógicos presentes noEduCatalog4RE. O processo de uso do catálogo será exemplificado através de um cenário fictício. A avaliação do catálogo teve caráter empírico e foi realizada por especialistas. Os resultados comprovam a utilidade do catálogo no desenvolvimento de SEs.

\section{Introdução}

Diversos estudos têm demonstrado que a utilização da Informática na Educação potencializa o processo de ensino e aprendizagem, a exemplo dos Softwares Educacionais. Porém, é necessário que eles contemplem as necessidades relacionadas as perspectivas pedagógicas, considerando os objetivos do professor. Diante disto, percebe-se que desenvolver softwares para educação não é trivial, pois eles necessitam de um conjunto de funcionalidades para atender aos aspectos pedagógicos [Giraffa, Marczak e Prikladnicki, 2005; Tchounikine, 2011].

Atualmente parece não existir um padrão de desenvolvimento para SEs e, consequentemente, a identificação de requisitos específicos a esse domínio pode ficar comprometida [Dalmon, Brandão e Brandão, 2012; Medeiros, 2012]. O trabalho de Cox e Bittencourt (2017), realizou uma revisão na literatura e percebeu que há uma carência em processos de desenvolvimento para Jogos Educacionais (JEs), que é um tipo de SE. Os que existem são adaptações de processos de desenvolvimento de softwares já existentes, como: cascata, espiral e RUP (Rational Unified Process). 
As teorias de aprendizagem explicam como as pessoas aprendem, e elas devem ser usadas explicitamente no desenvolvimento dos SEs [Lima, 2012], porém, sabe-se que os aspectos pedagógicos nem sempre são considerando durante o desenvolvimento dos SEs [Pietruchinski, 2011].

Diante do cenário apresentado, percebe-se, que são necessárias iniciativas que sistematizem o desenvolvimento dos SEs, considerando principalmente os aspectos pedagógicos. Existem na literatura alguns catálogos de requisitos específicos para mlearning (SE específico para celular), porém, eles não são ancorados em teorias de aprendizagem [Filho e Barbosa, 2012; Marçal et al., 2010; Economides, 2008]. O reuso de requisitos é visto como uma alternativa para auxiliar o desenvolvimento de SEs, pois ele torna a identificação dos requisitos mais sistemática, o que pode possibilitar um aumento na qualidade pedagógica do produto desenvolvido.

Este trabalho tem como objetivo apresentar o EduCatalog4RE, ele foi concebido para auxiliar o desenvolvimento de SEs na fase de elicitação de requisitos, por meio da técnica de reuso. O catálogo possui 62 requisitos pedagógicos fundamentados em teorias e/ou métodos de aprendizagem. Além disso, ele dispõe de 46 requisitos técnicos (gerais e usabilidade), que também foram identificados e incluídos no catálogo para auxiliar no desenvolvimento dos SEs. Para facilitar o uso do catálogo, foi sugerido um processo de uso composto por cinco fases, no qual é possível identificar os requisitos no catálogo através de palavras-chave, que estão associadas as teorias e métodos de aprendizagem (exemplos de palavras-chave: instrução programada e cotidiano do aluno).

Este artigo está organizado da seguinte forma. A seção 2 apresenta alguns trabalhos relacionados. A seção 3 descreve a metodologia usada no desenvolvimento e avaliação do EduCatalog4RE. A seção 4 detalha os 62 requisitos pedagógicos do EduCatalog4RE. O cenário fictício que mostra o processo de uso do catálogo, é descrito na subseção 4.1. A seção 5 mostra os resultados da avaliação e sugestões dos especialistas. Por fim, a seção 6 descreve as considerações finais e os trabalhos futuros.

\section{Trabalhos relacionados}

O trabalho de Filho e Barbosa (2013) realizou uma RSL para identificar características e requisitos para m-learning. Existem 44 requisitos, divididos em quatro critérios: técnico, educação, econômico e sócio-cultural. A avaliação dos requisitos por 36 especialistas. Além disso, os participantes classificaram os requisitos de acordo com nível de prioridade $((+)$ pouca relevância; $(++)$ relevante; e $(+++)$ muito relevante). Dois requisitos relacionados ao critério educacional são: pedagógico (interatividade) e usabilidade (ajuda, com nível de prioridade $(+++)$ muito relevante.

O trabalho de Marçal et al. (2010) apresenta um conjunto de requisitos para desenvolver aplicativos m-learning, em específico na área de matemática. Para a identificação dos requisitos foi realizado um estudo bibliográfico e um survey, porém, o trabalho não detalha o método utilizado. Os requisitos pedagógicos citados no trabalho de Marçal et al. (2012), fazem parte do EduCatalog4RE, são eles: apresentação e qualidade do conteúdo, organização do conteúdo, suporte e feedback ao aluno.

Outra proposta de requisitos para m-learning é apresentada no trabalho de Economides (2008). O trabalho também propõe a divisão dos requisitos em quatro dimensões: pedagógicos, sócio-cultural, econômico e técnico. Cada grupo apresenta um conjunto de requisitos. Os requisitos técnicos foram baseados no padrão de qualidade de software (ISO/IEC 9126), e os demais requisitos foram identificados através de 
VI Congresso Brasileiro de Informática na Educação (CBIE 2017)

Anais do XXVIII Simpósio Brasileiro de Informática na Educação (SBIE 2017)

pesquisas sobre teorias pedagógicas, aprendizagem adaptativa, usabilidade, interação humano-computador, aplicações móveis, padrões de qualidade e design de software.

$\mathrm{O}$ autor descreve cada requisito, mas não detalha sobre a sua implementação. Alguns exemplos de requisitos pedagógicos citados são: modelos de design instrucional, abrangência, apresentação, organização e qualidade do conteúdo, apoio e feedback ao estudante.

\subsection{Desenvolvimento do catálogo}

O EduCatalog4RE foi desenvolvido a partir de um estudo exploratório, configurando-se em uma RSL. O protocolo da revisão sistemática e os estudos primários identificados na revisão, podem ser vistos no trabalho de Henrique-Cunha et al. (2016).

\subsection{Avaliação do catálogo}

O método usado na avaliação é de natureza empírica, os participantes são especialistas em Informática na educação e de outras áreas (ex: engenharia de software, Neuropedagogia Computacional, redes de computadores, dentre outros). Foram utilizados dois questionários ${ }^{1}$ como instrumentos de coleta dos dados. A avaliação foi realizada em duas etapas, ou seja, foram geradas duas versões do EduCatalog4RE.

Os participantes da pesquisa foram selecionados através de eventos nacionais de Informática na educação (ex: CBIE), empresas que produzem Softwares educacionais (ex: Joystreet ${ }^{2}$, Guest $3 \mathrm{D}^{3}$ e Manifesto Games ${ }^{4}$ ), docentes de Universidades de vários estados brasileiros, grupos de pesquisa (ex: EducTec ${ }^{5}$, GINAPE - UFRJ ${ }^{6}$, InfoEduc Informática na educação (FURG) ${ }^{7}$, dentre outros).

Para responder às perguntas fechadas utilizou-se uma escala de cinco pontos do tipo Likert [Likert, 1932]. Sobre o tratamento dos dados, o aspecto quantitativo é baseado na estatística descritiva, a qual se preocupa em descrever os dados e transformá-los em informações. As assertivas utilizadas na avaliação do EduCatalog4RE foram avaliadas pela Média Aritmética Ponderada, com o objetivo de medir o grau de concordância dos participantes. Para realizar os cálculos estatísticos (desvio padrão, máximo, mínimo e mediana), utilizou-se a ferramenta SPSS Statistics Base $^{8}$ versão 22 . A escolha por esse software foi pela simplicidade da sua interface, e por ele atender as necessidades desta pesquisa. Em relação às questões abertas, optou-se por definir categorias (relevante, neutro e irrelevante) para as respostas dos participantes. A única categoria que altera o catálogo é a "relevante", as demais não são consideradas, pois não alteram o catálogo (neutro) ou não fazem parte escopo da pesquisa (irrelevante).

\section{EduCatalog4RE}

O EduCatalog4RE possui 108 requisitos, deste 62 são pedagógicos e 45 são gerais e de usabilidade, para facilitar a avaliação e o uso, foi desenvolvido um protótipo $\mathrm{Web}^{9}$. A Tabela 4 apresenta apenas os requisitos pedagógicos presentes no catálogo. Observe que para cada teoria ou método de aprendizagem, existem uma ou mais palavras-chave

\footnotetext{
Questionário 1: goo.gl/H1nN5m/ Questionário 2: goo.gl/Gr2dqE

${ }^{2}$ http://www.joystreet.com.br/

${ }^{3}$ http://www.guest3d.com.br/

${ }^{4} \mathrm{http}: / /$ www.manifestogames.com/

${ }^{5} \mathrm{https}: / /$ sites.google.com/site/edutecifes/pesquisadores

${ }^{6} \mathrm{http}: / /$ www.nce.ufrj.br/ginape/paginas/equipe.html

$7 \mathrm{http}$ ///infoeduc.c3.furg.br/index.php?Itemid=1826\&option=link\&id_site_componente=2796

${ }^{8} \mathrm{http}: / / \mathrm{www}-03 . \mathrm{ibm} . \mathrm{com} /$ software/products/pt/spss-stats-base

${ }^{9} \mathrm{http}: / /$ mgconsultoriaecursos.com.br/EduCatalog4RE/index.html
} 
VI Congresso Brasileiro de Informática na Educação (CBIE 2017)

Anais do XXVIII Simpósio Brasileiro de Informática na Educação (SBIE 2017)

associadas, e cada palavra-chave pode ter um ou mais requisitos associados a ela. Outro detalhe a destacar é que existem palavras-chave (com seus respectivos requisitos) que pertencem a mais de uma teoria ou método de aprendizagem. Por fim, foi possível identificar dois requisitos que contemplam todas as teorias e métodos de aprendizagem do EduCatalog4RE, as palavras-chave associadas são: qualidade pedagógica e acompanhar o desempenho dos alunos.

\section{Tabela 4. Requisitos pedagógicos do EduCatalog4RE baseadas em teorias e métodos de aprendizagem}

\begin{tabular}{|c|c|}
\hline $\begin{array}{c}\text { Aprendizagem } \\
\text { Significativa (ASG) }\end{array}$ & $\begin{array}{l}\text { Palavra-chave: Estrutura cognitiva } \\
\text { ASG1- Os conteúdos devem ser planejados de forma hierárquica e sequencial }\end{array}$ \\
\hline $\mathbf{A B P} \mathbf{P}^{10}$ & $\begin{array}{l}\text { Palavra-chave: Curto prazo } \\
\text { ABP1 - O sistema deve propor problemas menores com um curto prazo para resolução }\end{array}$ \\
\hline PBL $^{11}$ & $\begin{array}{l}\text { Palavra-chave: Grupo heterogêneo } \\
\text { PBL1- As atividades propostas pelo sistema devem considerar na divisão das equipes, } \\
\text { afinidades, competências e habilidades de cada aluno, para evitar concentração e/ou } \\
\text { escassez de competências e habilidades em uma mesma equipe. }\end{array}$ \\
\hline $\begin{array}{c}\text { ABPP } \\
(\mathbf{P B L}+\mathbf{A B P})\end{array}$ & Considerar todos os requisitos citados na PBL e ABP \\
\hline $\begin{array}{l}\text { PBL, ABP e ABPP } \\
\text { (RC_PAA) }\end{array}$ & $\begin{array}{l}\text { Palavra-chave: Situação problema } \\
\text { RC_PAA1- O sistema deve propor um ambiente que simule situações reais onde o } \\
\text { problema está inserido. }\end{array}$ \\
\hline Construtivismo (CONS) & $\begin{array}{l}\text { Palavra-chave: Construção do conhecimento } \\
\text { CONS1 - Exibir feedback construtivo } \\
\text { CONS2 - Exibir feedback individualizado } \\
\text { CONS3- O sistema deve possuir vários desafios, pois esse requisito de jogabilidade é } \\
\text { importante para motivar os usuários. } \\
\text { CONS4 - Propor reflexões críticas ao trabalhar os conteúdos } \\
\text { Palavra-chave: Autonomia do aprendiz } \\
\text { CONS5 - O sistema deve permitir que o usuário realize escolhas durante a interação } \\
\text { CONS6 - O sistema deve propor ao usuário construir o máximo de soluções possíveis. }\end{array}$ \\
\hline $\begin{array}{l}\text { Construcionismo } \\
\text { (CON) }\end{array}$ & $\begin{array}{l}\text { Palavra-chave: Criar desafios } \\
\text { CON1- O sistema deve permitir que o usuário crie desafios para outros jogadores }\end{array}$ \\
\hline $\begin{array}{l}\text { Taxonomia de Bloom } \\
\text { (TX) }\end{array}$ & $\begin{array}{l}\text { Palavra-chave: Níveis cognitivos } \\
\text { TX1- Os conteúdos devem ser divididos em vários níveis de conhecimento, iniciando } \\
\text { sempre do menor nível de complexidade. }\end{array}$ \\
\hline $\begin{array}{c}\text { Teoria da } \\
\text { Aprendizagem } \\
\text { Multimídia (TAM) }\end{array}$ & $\begin{array}{l}\text { Palavra-chave: Recursos multimídia } \\
\text { TAM1- Prover recursos que permitam que diferentes canais de processamento do } \\
\text { usuário venham ser estimulados durante a aprendizagem. }\end{array}$ \\
\hline $\begin{array}{l}\text { ACT (Atomic } \\
\text { Components } \\
\text { of Thought) } \\
\quad(\text { ACT) }\end{array}$ & $\begin{array}{l}\text { Palavra-chave: Mapear o comportamento } \\
\text { ACT1- O sistema deve acompanhar os alunos durante a sua interação no ambiente. } \\
\text { Palavra-chave: Baseado em regras } \\
\text { ACT2- O sistema deve ser baseado em regras (condição e ação) } \\
\text { Palavra-chave: Plano de ensino } \\
\text { ACT3- Os planos de ensino devem ser baseados no domínio do aluno inferido sobre os } \\
\text { conhecimentos abordados. Portanto, o sistema deve abordar conteúdos que sejam do } \\
\text { domínio do aluno. }\end{array}$ \\
\hline
\end{tabular}

\footnotetext{
${ }^{10}$ Aprendizagem Baseada e Problemas e por Projetos

${ }^{11}$ (Problem-Based Learning)
} 
VI Congresso Brasileiro de Informática na Educação (CBIE 2017)

Anais do XXVIII Simpósio Brasileiro de Informática na Educação (SBIE 2017)

\begin{tabular}{|c|c|c|}
\hline $\begin{array}{c}\text { Comportamentalismo } \\
\text { (COMP) }\end{array}$ & \multicolumn{2}{|c|}{$\begin{array}{l}\text { Palavra-chave: Instrução programada } \\
\text { COMP1- Organizar e disponibilizar os conteúdos (desafios e aulas) em ordem } \\
\text { cronológica. } \\
\text { COMP2 - O software deve penalizar o usuário, que serão consequências das suas ações. } \\
\text { COMP3 - O sistema deve prover feedback imediato para o usuário de acordo com as } \\
\text { suas ações. } \\
\text { COMP4 - O sistema deve beneficiar o usuário caso ele acerte os desafios ou pegue itens } \\
\text { importantes no cenário. }\end{array}$} \\
\hline $\begin{array}{l}\text { Sócio-Interacionismo } \\
\text { (SOC) }\end{array}$ & $\begin{array}{l}\text { Palavra-chave: Interação com o software } \\
\text { A descrição dos requisitos SOC1 até o } \\
\text { SOC20 e SOC28 é: a funcionalidade "X" } \\
\text { deve permitir interação do usuário com o } \\
\text { sistema. } \\
\text { SOC1- "Abrir" } \\
\text { SOC2- "Acessar" } \\
\text { SOC3-"Agendar" } \\
\text { SOC4- "Alterar" } \\
\text { SOC5-"Analisar/Avaliar" } \\
\text { SOC6- "Adicionar" } \\
\text { SOC7-"Atribuir" } \\
\text { SOC8-"Atualizar" } \\
\text { SOC9-"Cadastrar" } \\
\text { SOC10-"Compartilhar" SOC11- Configurar } \\
\text { SOC12- Consultar/pesquisar } \\
\text { SOC13- "Copiar" } \\
\text { SOC14- "Comentar" } \\
\text { SOC15- "Enviar" } \\
\text { SOC16- "Executar" } \\
\text { SOC17- "Excluir" } \\
\text { SOC18-"Exibir/visualizar" } \\
\text { SOC19- "Gerenciar" } \\
\text { SOC20- "Listar" } \\
\text { SOC28- Salvar }\end{array}$ & $\begin{array}{l}\text { SOC21 - Os objetos do ambiente devem } \\
\text { mudar de estado quando o usuário } \\
\text { interagir com eles, desta forma o usuário } \\
\text { terá uma percepção maior durante a } \\
\text { interação. } \\
\text { SOC22- Os objetos do ambiente devem } \\
\text { ser manipuláveis, dessa forma o usuário } \\
\text { poderá ter maior interação e se sentirá } \\
\text { mais imerso no ambiente. } \\
\text { SOC23- O sistema deve perceber as } \\
\text { possíveis dificuldades do usuário e } \\
\text { oferecer ajuda, através das interaços do } \\
\text { usuário com outros personagens. Além } \\
\text { disso, os personagens podem desafiar os } \\
\text { usuários, a exemplo dos inimigos. } \\
\text { SOC24- O sistema deve permitir que o } \\
\text { usuário possa acessar informações } \\
\text { externas ou internas. } \\
\text { SOC25 - Os personagens devem } \\
\text { interagir entre si e com objetos } \\
\text { distribuídos pelo ambiente. } \\
\text { SOC26 - A funcionalidade "Download" } \\
\text { deve permitir interação do usuário com o } \\
\text { sistema. } \\
\text { SOC27 - O sistema deve permitir que o } \\
\text { usuário possa interagir no ambiente de } \\
\text { várias formas (ex: mouse, zoom e } \\
\text { teclado). }\end{array}$ \\
\hline $\begin{array}{l}\text { Teoria do conhecimento } \\
\text { (TC) }\end{array}$ & \multicolumn{2}{|c|}{$\begin{array}{l}\text { Palavra-chave: Notificação sonora } \\
\text { TC1- O sistema deve propor feedback sonoro para auxiliar os usuários, principalmente } \\
\text { aqueles que não são alfabetizados }\end{array}$} \\
\hline $\begin{array}{l}\text { Teoria das Inteligências } \\
\text { Múltiplas (TIM) }\end{array}$ & \multicolumn{2}{|c|}{$\begin{array}{l}\text { Palavra-chave: Perfil do usuário } \\
\text { TIM1- O sistema deve ser capaz de detectar o perfil do usuário, isso deve acontecer } \\
\text { antes dele começar a interagir com o sistema. } \\
\text { Palavra-chave: Sistema adaptativo } \\
\text { TIM2-O sistema deve se adaptar as características do usuário. } \\
\text { Palavra-chave: Personalidade e sentimento do usuário } \\
\text { TIM3- O sistema deve levar em consideração a personalidade e/ou sentimento do } \\
\text { usuário, para propor atividades que considerem os estilos de aprendizagem. }\end{array}$} \\
\hline \multicolumn{3}{|c|}{ Requisitos que compartilham mais de uma teoria e/ou método de aprendizagem } \\
\hline $\begin{array}{l}\text { Construtivismo + } \\
\text { Aprendizagem } \\
\text { significativa } \\
\text { (RC_CA) }\end{array}$ & \multicolumn{2}{|c|}{$\begin{array}{l}\text { Palavra-chave: Adaptável ao nível do aprendiz } \\
\text { RC_CA1- O conteúdo proposto deve se relacionar com os conhecimentos prévios do } \\
\text { aluno }\end{array}$} \\
\hline $\begin{array}{l}\text { Construtivismo + Sócio } \\
\text { interacionismo + ABP } \\
\quad(\text { RC_CSA })\end{array}$ & \multicolumn{2}{|c|}{$\begin{array}{l}\text { Palavra-chave: Cooperação/Colaboração } \\
\text { RC_CSA1- O software deve possuir atividades que proporcionem cooperação entre as } \\
\text { pessoas durante a resolução dos projetos. }\end{array}$} \\
\hline
\end{tabular}


VI Congresso Brasileiro de Informática na Educação (CBIE 2017)

Anais do XXVIII Simpósio Brasileiro de Informática na Educação (SBIE 2017)

\begin{tabular}{|c|c|}
\hline $\begin{array}{c}\text { Construtivismo + sócio } \\
\text { interacionismo } \\
\text { (RS_CS) }\end{array}$ & $\begin{array}{l}\text { Palavra-chave: Interação social } \\
\text { RC_CS1- Disponibilizar o trabalho em rede (Internet) } \\
\text { RC_CS2- Promover interações e estimular o trabalho em grupo. } \\
\text { RC_CS3- Promover interação entre os usuários e entre o usuário e o sistema, através de } \\
\text { diversas ferramentas de comunicação. } \\
\text { RC_CS4- O sistema deve disponibilizar um espaço para interação entre os usuários. }\end{array}$ \\
\hline $\begin{array}{l}\text { Teoria do } \\
\text { conhecimento+ } \\
\text { Aprendizagem } \\
\text { significativa + sócio- } \\
\text { interacionismo } \\
\text { (TCA) }\end{array}$ & $\begin{array}{l}\text { Palavra-chave: Cotidiano do aluno } \\
\text { TCA1- O sistema deve possuir objetos do cotidiano, desta forma o usuário irá se } \\
\text { familiarizar com o ambiente, e a aprendizagem terá mais sentido para o aprendiz. } \\
\text { TCA2- É preciso criar um ambiente no qual os símbolos e signos do cotidiano dos } \\
\text { alunos sejam transcritos visualmente. }\end{array}$ \\
\hline \multicolumn{2}{|c|}{ Requisitos que são comuns a todas as teorias e métodos de aprendizagem existentes no EduCatalog4RE } \\
\hline \multicolumn{2}{|c|}{$\begin{array}{l}\text { Palavra-chave: Qualidade pedagógica } \\
\text { RCT1- O conteúdo e recursos disponibilizados devem ter qualidade pedagógica, ou seja, eles devem atender aos } \\
\text { objetivos do professor e se adequar ao público alvo. } \\
\text { Palavra-chave: Acompanhar desempenho dos alunos } \\
\text { RCT2- O sistema deve permitir que o usuário (professor) avalie o desempenho do usuário (aluno), através dos } \\
\text { artefatos gerados e/ou respostas dos exercícios (desafios). }\end{array}$} \\
\hline
\end{tabular}

\subsection{Sugestão de uso}

A sugestão de uso do catálogo é composta por cinco fases, a seguir será detalhada cada uma delas:

$1^{a}$ fase - Um determinado usuário (Ex: engenheiro de requisitos ou analista) deverá compreender as necessidades dos stakeholders, ao final deve-se gerar uma lista de necessidades.

$2^{\mathbf{a}}$ fase - A lista de necessidades irá auxiliar na escolha dos tipos de requisitos (pedagógico, usabilidade e gerais).

$3^{\mathbf{a}}$ fase - Nesta fase é desejável que uma equipe multidisciplinar (psicólogo, pedagogo, dentre outros) participe da escolha dos requisitos. Principalmente em relação a escolha dos requisitos pedagógicos. Portanto, é necessário ter em mãos a lista de necessidades dos stakeholders, já com a escolha sobre o tipo do requisito. Após isso, é realizada a escolha das palavras-chave (ex: Construtivismo possui cinco palavraschaves, um exemplo é: construção do conhecimento). Por fim, é gerada uma lista de palavras-chave para cada tipo de requisito. As palavras-chave foram sugeridas para facilitar a busca pelos requisitos, o trabalho de $\mathrm{Wu}$ et al. [2012] foi utilizado como referência.

$4^{\text {a }}$ fase - Após obter-se a lista de palavras-chave, é necessário que o usuário selecione e analise os requisitos associados a elas. Por fim, é gerada a lista de requisitos selecionados.

$5^{\text {a }}$ fase - Os requisitos selecionados na fase anterior devem ser refinados em funcionalidades implementáveis. Vale enfatizar que esse trabalho não contempla esta etapa, pois não foi possível identificar nos estudos primários esse nível de detalhes.

Para demonstrar o processo de uso do catálogo, um cenário fictício foi elaborado. A Tabela 5 apresenta como seria o resultado em cada fase do processo, vale salientar que este trabalho só contempla até a quarta fase. A quinta fase não está no escopo deste trabalho, pois se faz necessário utilizar o catálogo em cenários reais de desenvolvimento de SE, para conseguir transformar os requisitos identificados em funcionalidades implementáveis. 
VI Congresso Brasileiro de Informática na Educação (CBIE 2017)

Anais do XXVIII Simpósio Brasileiro de Informática na Educação (SBIE 2017)

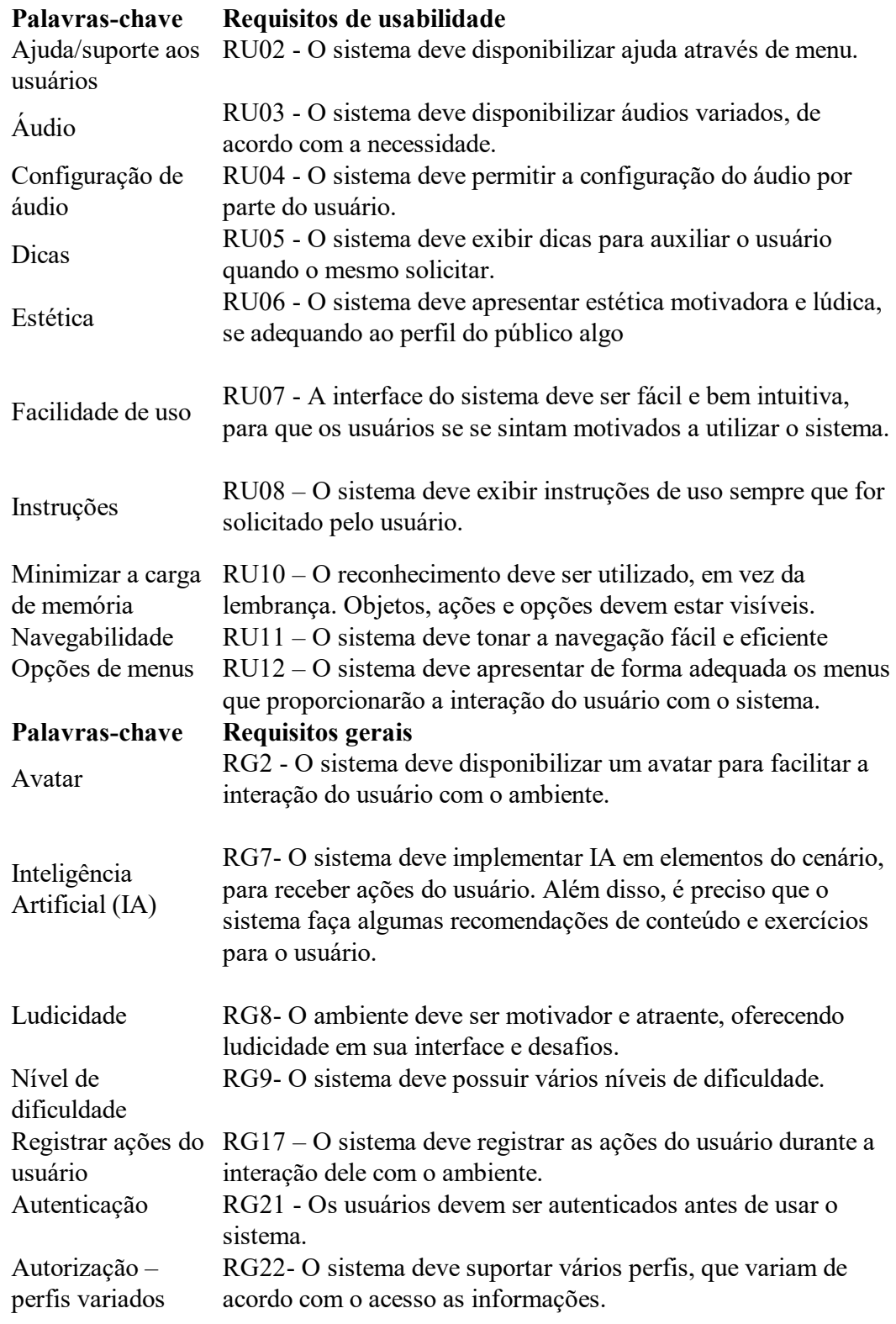

\section{Análise da avaliação}

A avaliação da primeira versão do EduCatalog4RE foi de natureza qualitativa, o objetivo foi receber os feedbacks fornecidos pelos especialistas em relação aos requisitos do catálogo, principalmente os pedagógicos. A amostra foi composta de 29 participantes, dos quais 68,97\% possuíam experiência com o desenvolvimento de SEs. Os feedbacks dos especialistas foram classificados em três categorias (relevante, neutro e irrelevante), porém, apenas a categoria "relevante" foi considerada para realizar alteração do catálogo. Algumas modificações realizadas foram: retirar termos específicos para tornar o requisito genérico, desenvolver uma sugestão de uso do catálogo, uniformizar a escrita dos requisitos, excluir requisitos semelhantes, inserir identificadores (de acordo com o tipo do requisito, e teoria e método de aprendizagem) nos requisitos, dentre outros. Após isso foi gerada a segunda versão do catálogo. 
A avaliação da segunda versão do EduCatalog4RE foi de natureza quantitativa, o objetivo foi verificar a aceitação dos participantes em relação aos seguintes aspectos: utilidade do catálogo na identificação dos requisitos, uso de palavras-chave para auxiliar a identificação dos requisitos, qualidade e quantidade dos requisitos, dentre outros. A amostra contemplou 23 participantes, foi comprovado que $61 \%$ deles avaliaram a primeira versão do EduCatalog4RE. Os resultados mostraram que $98 \%$ dos participantes acham útil usar o catálogo na identificação de requisitos para SEs. Em relação a facilidade de usar o catálogo, $65 \%$ dos participantes se posicionaram positivamente. As demais assertivas comprovam a aceitação dos especialistas em relação ao processo de sugestão de uso (65\%), qualidade dos requisitos $(69,5 \%)$, quantidade dos requisitos (61\%), dentre outros. Após a análise geral dos resultados, observa-se que a maioria dos participantes optou pela alternativa de concordância (4 e 5 na escala likert). Diante dos resultados, foi possível observar que os participantes concordaram que o EduCatalog4RE é uma ferramenta viável na identificação de requisitos para SEs. Algumas sugestões dos especialistas sobre o EduCatalog4RE, são apresentadas na Tabela 6 . Todas as sugestões podem ser vistas no trabalho de HenriqueCunha et al. (2016).

Tabela 6. Sugestões dos especialistas na $2^{\mathrm{a}}$ avaliação do EduCatalog4RE

\begin{tabular}{|c|l|c|}
\hline Participantes (P) & \multicolumn{1}{|c|}{ Descrição das respostas } & Categoria \\
\hline P15 & $\begin{array}{l}\text { Ao auxiliar na identificação de requisitos, o EduCatalog4RE também pode } \\
\text { ser visto como um instrumento interessante para promover a análise e } \\
\text { comparação de funcionalidades entre diferentes softwares educacionais. }\end{array}$ & $\begin{array}{c}\text { Relevante } \\
\text { (Trabalhos } \\
\text { futuros) }\end{array}$ \\
\hline P17 & $\begin{array}{l}\text { Uma sugestão é incluir a faixa etária do público-alvo como critério para } \\
\text { análise dos requisitos, pois isso também pode influenciar nas decisões de } \\
\text { desenvolvimento. }\end{array}$ & $\begin{array}{c}\text { Relevante } \\
\text { (Trabalhos } \\
\text { futuros) }\end{array}$ \\
\hline
\end{tabular}

Vários aspectos foram avaliados em ambas as versões, como: estrutura do catálogo (inclui vários aspectos como: escrita e clareza dos requisitos, disposição dos requisitos no catálogo, dentre outros), classificação dos tipos de requisitos, correspondência dos requisitos pedagógicos às suas respectivas teorias de aprendizagem, definição e clareza dos requisitos de usabilidade, sugestão do processo de desenvolvimento, uso de palavras-chave para auxiliar a identificação de requisitos, dentre outros.

\section{Considerações finais}

Diversos trabalhos ressaltam a ausência de processos sistemáticos para desenvolver SEs, afirmando que eles geralmente são desenvolvidos de forma ad hoc [Gomes e Wanderley, 2003], o que pode comprometer a sua qualidade pedagógica [Dalmon, Brandão e Brandão, 2012; Medeiros, 2012]. Sem um processo sistemático, a identificação dos requisitos pode ficar comprometida, principalmente porque eles devem atender aos aspectos pedagógicos. Nesse cenário, o reuso de requisitos é visto como uma alternativa que é capaz de sistematizar o desenvolvimento de SEs, o que possibilita um aumento na qualidade pedagógica do produto final.

O presente trabalho apresentou o EduCatalog4RE, que é uma alternativa viável para a sistematizar o desenvolvimento de SES, através da técnica de reuso. O catálogo possui 62 requisitos pedagógicos, eles são fundamentados em seis teorias e sete métodos de aprendizagem. Além disso, um processo de uso foi sugerido para auxiliar a compreensão dos usuários na identificação dos requisitos. Após a avaliação dos especialistas foi possível melhorar o catálogo em vários aspectos, a exemplo da escrita e clareza dos requisitos. 
VI Congresso Brasileiro de Informática na Educação (CBIE 2017)

Anais do XXVIII Simpósio Brasileiro de Informática na Educação (SBIE 2017)

Como trabalhos futuros, pretende-se desenvolver uma nova versão do EduCatalog4RE, levando em consideração as sugestões dos especialistas que participaram da segunda avaliação do EduCatalog4RE.

\section{Referências}

Cox, K. K., Bittencourt, R. A. (2017) Estudo Bibliográfico sobre o Processo de Construção de Jogos Digitais: A Necessidade de Sinergia entre o Educar e o Divertir. Revista Brasileira de Informática na Educação - RBIE, V.25, N.1.

Dalmon, D. L., Brandão, A. A., Brandão, L. O. (2012). Uso de métodos e técnicas para desenvolvimento de software educacional em universidades brasileiras. In: I Workshop de Desafios da Computação Aplicada à Educação-DesafIE. Anais. Curitiba, Paraná, p.236-245, 2012.

Economides, A. A. (2008) Requirements of Mobile Learning Applications. International Journal of Innovation and Learning, v. 5, n. 5, p.457-479, 2008.

Filho, N.F.D.; Barbosa, F. A Requirements Catalog for Mobile Learning Environments. In: Proceedings of the 28th Annual ACM Symposium on Applied Computing (SAC '13). Anais. New York, NY, USA, 2013, p.1266-1271.

Giraffa, L., Marczak, S., Prikladnicki, R. PDS-E: Em direção a um processo para desenvolvimento de Software Educacional. In: Workshop de Informática na Escola. Anais. São Leopoldo, Rio Grande do Sul, 2005, p.2833-2841.

Gomes, A. S., Wanderley, E. G. Elicitando requisitos em projetos de Software Educativo. In: Workshop de Informática Educativa. Anais. [S.I], 2003. p. 119-130.

Henrique, M. S., Silva, C. T. L. L., da Silva, D. R. D., e Tedesco, P. C. A. R. (2015). Uma Revisão Sistemática da Literatura sobre o uso de Teorias de Aprendizagem em Softwares Educacionais. RENOTE, v. 13, n. 2, 2015.

Lima, F. A. Métodos, Técnicas e Ferramentas para o Desenvolvimento de Software Educacional: Um Mapeamento Sistemático. Dissertação (Mestrado) - Universidade Federal de Pernambuco, Recife, 2012.

Marçal, E., de Lima, L., Júnior, M., Viana, W., Andrade, R., e Ribeiro, J. W. (2010). Da elicitação de requisitos ao desenvolvimento de aplicações de mobile learning em matemática. In: Anais do Simpósio Brasileiro de Informática na Educação-SBIE. 2010.

Medeiros, A. F. Elicitação de critérios essenciais para a adaptação de uma metodologia ágil para o desenvolvimento de software educativo. Trabalho de Conclusão de curso (Graduação). Universidade Estadual da Paraíba, Patos, 2012.

Pietruchinski, M. H. et al. (2011). Os jogos educativos no contexto do SBIE: uma revisão sistemática de Literatura. In: Simpósio Brasileiro de Informática na EducaçãoSBIE.

Tchounikine, P. Computer Science and Educational Software Design: A Resource for Multidisciplinary Work in Technology Enhanced Learning/Pierre Tchounikine. Springer, 2011.

$\mathrm{Wu}, \mathrm{W}$.-H. et al. Investigating the learning-theory foundations of game-based learning: a meta-analysis. Journal of Computer Assisted Learning, v. 28, n. 3, p. 265-279, 2012. 